\title{
Silencing Aurora-A with siRNA inhibits cell proliferation in human lung adenocarcinoma cells
}

\author{
NING ZHONG ${ }^{1 *}$, SHUNBIN SHI $^{2 *}$, HONGZHEN WANG $^{3 *}$, GUANGZHOU WU ${ }^{4 *}$, YUNLIANG WANG ${ }^{5}$, \\ QIANG MA ${ }^{6}$, HONGWEI WANG $^{7}$, YUANHUA LIU $^{8}$ and JINZHI WANG ${ }^{9}$ \\ ${ }^{1}$ Department of Thoracic Surgery, Kunshan First People's Hospital Affiliated to Jiangsu University, \\ Kunshan; ${ }^{2}$ Department of Thoracic Surgery, Affiliated Wujiang Hospital of Nantong Uinversity, Wujiang; \\ ${ }^{3}$ Department of Orthopaedics, the First People's Hospital of Kunshan City, Kunshan; ${ }^{4}$ Department of Thoracic Surgery, \\ Yancheng First People's Hospital Affiliated to Jiangsu University, Yancheng, Jiangsu; ${ }^{5}$ Department of Neurology, \\ The 148 Central Hospital of PLA, Zibo City, Shangdong; ${ }^{6}$ State Key Laboratory of Organ Failure Research, \\ Institute of Antibody Engineering, Southern Medical University, Guangzhou, Guangdong, P.R. China; \\ ${ }^{7}$ Department of Medicine, The University of Chicago, Chicago, IL, USA; ${ }^{8}$ Department of Chemotherapy, \\ the Affiliated Jiangsu Cancer Hospital of Nanjing Medical University and Jiangsu Institute of Cancer Research, \\ Nanjing; ${ }^{9}$ Department of Cell Biology, School of Medicine, Soochow University, Suzhou, Jiangsu, P.R. China
}

Received February 15, 2016; Accepted April 20, 2016

DOI: 10.3892/ijo.2016.3605

\begin{abstract}
Aurora kinase A (AURKA) is an oncogenic serine/ threonine kinase, it plays important roles in tumorigenesis and chemoresistance. In this study, we investigated the expression of AURKA in lung adenocarcinoma tissues, the role of small interference RNA targeting AURKA on growth, cell cycle, and apoptosis of lung adenocarcinoma cell lines in vitro. The AURKA is highly expressed in lung adenocarcinoma tissues and human lung adenocarcinoma cell lines. Lentivirusmediated short hairpin RNA (shRNA) was used to knock down AURKA expression in human lung adenocarcinoma cell lines H1299 and A549. The results indicated that depletion of AURKA could inhibit cell growth, cause cell cycle arrest and apoptosis. The potential mechanisms of AURKA inhibition induced cell cycle arrest and apoptosis are associated with downregulated RAF-1, CCND2, CCND3, CDK4, PAK4, EGFR and upregulated WEE1 expression. Furthermore, AURKA knockdown cooperated with vincristine (VCR) to
\end{abstract}

Correspondence to: Dr Jinzhi Wang, Department of Cell Biology, School of Medicine, Soochow University, Suzhou, Jiangsu 215123, P.R. China

E-mail: 451588568@qq.com

Dr Yuanhua Liu, Department of Chemotherapy, the Affiliated Jiangsu Cancer Hospital of Nanjing Medical University and Jiangsu Institute of Cancer Research, Nanjing 210009, P.R. China

E-mail: yuanhualiu@hotmail.com

*Contributed equally

Key words: lentivirus, RNA interference, Aurora-A, lung adenocarcinoma cells, vincristine repress A549 cell proliferation. Therefore, AURKA plays important roles in the proliferation of human lung adenocarcinoma cells, which suggests that AURKA could be a promising tool for lung adenocarcinoma therapy.

\section{Introduction}

Lung carcinoma, the most common type of tumor, is currently the leading cause of tumor-related deaths world-wide. Lung cancer is a bronchogenic carcinoma and histologically subdivided into small cell lung cancer (SCLC) and non-small cell lung cancers (NSCLC). Lung adenocarcinoma, the most common subtype of NSCLC, is the most prevalent pathological form of lung cancer (1-3). Although surgical therapy, chemotherapy, radiation therapy and targeted therapy have made significant progress in recent years, the prognosis of lung adenocarcinoma is still poor (4). Thus, a more detailed understanding of the key biomarkers and molecular mechanisms of initiation, development and progression of lung adenocarcinoma is extremely important for improving the diagnosis, prevention and treatment of this disease $(5,6)$.

The Aurora kinases family is comprised of three members: AURK-A, -B and -C. These kinase members are key regulators of mitosis and multiple signaling pathways. Human AURKA gene maps to chromosome 20q13.2, and is more extensively studied, especially in tumor fields (7). AURKA functions as an oncogene and overexpressed in several kinds of cancer including malignancies breast, and colon cancers, as well as in neuroblastoma $(7,8)$. Due to essential roles of AURKA in mitotic entry, DNA damage checkpoint recovery and centrosome and spindle maturation, the inhibition of AURKA expression is a promising therapeutic for multiple cancers. With the development of AURKA inhibitors, several clinical trials using AURKA inhibitors in multiple tumor types have been applied, especially Alisertib, a potent and selective inhibitor currently 
in phase III. The results of AURKA inhibitors from clinical trials indicated the complexity of such treatment in cancers, which depends on many factors. To select those patients that better react to AURKA inhibitors, and test the cooperative effect of AURKA inhibitors with different antitumoral drugs should be explored in future studies $(7,9,10)$. RNA interference (RNAi), the process of sequence-specific post-transcriptional gene silencing, is a revolutionary tool for the analysis of gene function and gene therapy for cancer and other diseases (11).

To our knowledge, this is the first study associated with the relationship between AURKA and lung adenocarcinoma. We confirmed that AURKA is highly expressed in lung adenocarcinoma tissues and human lung adenocarcinoma cell lines. Furthermore, we found that knockdown of AURKA in human lung adenocarcinoma could inhibit cell growth and proliferation in vitro. Importantly, AURKA has cooperative effects with VCR on suppressing human lung adenocarcinoma proliferation. Therefore, our results provide novel insights into AURKA as a therapeutic target for lung adenocarcinoma.

\section{Materials and methods}

Main reagents. Mouse anti-human AURKA polyclonal antibody was purchased from ProteinTech Group (Wuhan, Hubei, China); mouse anti-human AURKA monoclonal antibody was purchased from Abcam (Abcam, Cambridge, UK); Taq DNA polymerase was obtained from Fermentas, Inc. (Waltham, MA, USA); Lipofectamine 2000, Opti-MEM and the SuperScript III reverse transcriptase (RT) kit were purchased from Invitrogen (Carlsbad, CA, USA); Glyceraldehyde 3-phosphate dehydrogenase (GAPDH) and bovine serum albumin were purchased from Sigma Chemical Co. (St. Louis, MO, USA); VCR were purchased from Shenzhen Wan Le Pharmaceutical Co., Ltd. (Shenzhen, Guangdong, China); cell culture media, fetal bovine serum (FBS) and other supplementary materials were purchased from Gibco Co. (Grand Island, NY, USA).

Cell culture. Human lung adenocarcinoma H1299 cells and A549 cells were obtained from the Cell Bank of Chinese Academy of Sciences (Shanghai, China) in 2013 and were identified by STR (short tandem repeat) method in 2014. All cells were cultured in DMEM (Dulbecco's modified Eagle's medium) (Gibco Co.) supplemented with $10 \%$ fetal calf serum (FCS) and grown in a humidified incubator at $37^{\circ} \mathrm{C}$ and $5 \%$ $\mathrm{CO}_{2}$.

Tissue collection. From January 2005 to January 2010, 101 patients with lung adenocarcinoma underwent resection in the Kunshan First People's Hospital affiliated to Jiangsu University. All cases of lung adenocarcinoma were clinically and pathologically proven. This study was approved by the medical ethics committee of the Kunshan First People's Hospital with the reference number: 10. All participants have provided verbal informed consent to participate in this study. We recorded participant consents through the telephone communication. This consent procedure was approved by the ethics committees.

Immunohistochemical detection of AURKA in lung adenocarcinoma tissues. Immunohistochemistry (IHC) studies were performed according to the manufacturer's instructions. Briefly, $3 \mu \mathrm{m}$-thick sections was deparaffinized and rehydrated, then incubated in 3\% hydrogen peroxide for 15 min to block endogenous peroxidase activity. These tissue slides were boiled in EDTA buffer ( $\mathrm{pH} 9.0$ ) for $10 \mathrm{~min}$ for antigen retrieval. At room temperature, $10 \%$ normal rabbit serum was introduced for blocking non-specific binding. At $4^{\circ} \mathrm{C}$ refrigerator, the slides was incubated with polyclonal antibody against AURKA at a dilution of 1:100 in PBS for $1 \mathrm{~h}$, rinsed five times with PBS, they were incubated with goat anti-mouse IgG conjugated with horseradish peroxidase for $30 \mathrm{~min}$ at room temperature. The histologic sections were developed with DAB (3,3'-diaminobenzidine-tetrahydrochloride-dihydrate) and lightly counterstained with haematoxylin. The histologic sections were read using light microscopy.

Each case was scored according to the percentage of positive cells to total cancer cells and the staining intensity of the positive cells. Regarding cell counting under microscope, at least 10 high-power fields were randomly selected. The area of staining was divided into four levels as follows: no staining of cells in any microscopic fields was scored $0 ;<30 \%$ of tissue stained positive was scored 1; between 30 and $60 \%$ stained positive was scored $2 ;>60 \%$ stained positive was scored 3 . In each slice, no staining, weak staining, moderate staining and strong staining were scored as $0,1,2$, and 3 , respectively.

AURKA expression and prognosis. A total of 443 samples from 4 institutions [Moffitt Cancer Center (HLM), University of Michigan Cancer Center (UM), the Dana-Farber Cancer Institute (DFCI) and Memorial Sloan-Kettering Cancer Center (MSK)] were used to investigate the association of the AURKA expression and prognosis in lung adenocarcinoma, as previously published (12). Patients were separated into high or low AURKA expression groups based on the first quantile (25\%) of the AURKA expression values of total samples. KaplanMeier survival analysis was used to estimate survival curves and difference between curves was evaluated by log-rank test. Multivariate Cox proportional hazards regression with covariate age, gender, and stage was carried out to measure the independent prognostic factors. All tests were two-tailed and $\mathrm{p}<0.05$ were considered significant.

RT-PCR (Reverse transcription PCR) analysis. The TRIzol reagent (Invitrogen) following the protocol of the manufacturer (Invitrogen). RNA (1 $\mu \mathrm{g}$ ) was subjected to reverse transcription. The PCR primers used were as follows: for AURKA: forward 5'-GCCCTGTCTTACTGTCATTCG-3' and reverse 5'-AGGTCTCTTGGTATGTGTTTGC-3'; for GAPDH: forward 5-TGACTTCAACAGCGACACCCA-3' and reverse 5'-CACCCTGTTGCTGTAGCCAAA-3'; PCR products were separated by electrophoresis in $1 \%$ agarose gel, visualized by staining with ethidium bromide and photographed under ultraviolet light.

Recombination lentivirus generation and cell infection. The human AURKA-specific small interfering RNA (siRNA) sequence is 5'-GAAAGCTCCACATCAATAA-3', designed with an online software of Invitrogen using AURKA sequence (GeneBank code: NM_003600) as a reference. The nonsilencing (NS) sequence (5'-TTCTCCGAACGTGTCACGT-3') 
was used as scrambled control that has been widely used (13). The short hairpin RNA (shRNA) cassette against AURKA is 5'-CCGGCAGAAAGCTCCACATCAATAATTCAAGAGA TTATTGATGTGGAGCTTTCTGTTTTTG-3', with two cohesive ends for ligation into the pGCSIL-GFP vector. The double stranded shRNA oligonucleotide were ligated into pGCSIL-GFP vector linearized by restriction enzyme EcoRI and AgeI.

Next, lentiviral vector that expressed the AURKA-specific siRNA or negative control siRNA, together with pHelper 1.0 and pHelper 2.0 plasmids were co-transfected into HEK293T cells with Lipofectamine 2000 for lentivirus generation, according to the manufacturer's instructions (Invitrogen). After $48 \mathrm{~h}$ of transfection, the lentiviral particles were harvested and purified with ultracentrifugation. Due to the produced lentiviruses carrying green fluorescence protein (GFP), the viral titer was determined by counting green cells with serial dilutions under fluorescence microscopy at 5 days after infection. For lentivirus infection, H1299 and A549 cells were grown in 6-well plates at 70-80\% confluence and infected with AURKAspecific siRNA lentivirus or control lentivirus at MOI of 20. Five days after infection, cells expressing GFP protein were observed using fluorescence microscopy to determine the infection efficiency. There were two experimental groups for each cell line: LV-AURKA infected cells (AURKA-siRNA) and LV-NS infected cells (scr-siRNA).

Real-time PCR analysis. Total RNA was initially extracted from cultured lung adenocarcinoma cells using TRIzol (Invitrogen) and treated with RNase-free DNase I. Standard reverse transcription reaction was performed using a Promega M-MLV cDNA synthesis kit following the manufacturer's instructions. The real-time reverse transcription polymerase chain reaction was performed using the SYBR Green One-Step qRT-PCR kit (Invitrogen) according to the kit's procedure manual. GAPDH was used as an internal control. The PCR primers used were: AURKA: forward 5'-GCCCTGTCTTACT GTCATTCG-3', AURKA: reverse 5'-AGGTCTCTTGGTAT GTGTTTGC-3'; GAPDH: forward 5'-TGACTTCAACAGC GACACCCA-3', and GAPDH: reverse 5'-CACCCTGTTGCT GTAGCCAAA-3. The relative gene expression levels were calculated using the $2^{-\Delta \Delta C T}$ algorithm.

Cellomics to test inhibition of lung adenocarcinoma cell proliferation following knockdown of AURKA. The monolayer culture growth rate was determined by using a Cellomics Arrayscan (Thermo Fisher Scientific Inc., Waltham, MA, USA). Briefly, after the lung adenocarcinoma H1299 cells were infected with virus for 3 days, cells were seeded into 96-well plates and cultured in a humidified atmosphere of 5\% $\mathrm{CO}_{2}$ at $37^{\circ} \mathrm{C}$. The Cell viability was measured at $0,1,2,3$, 4 and 5 days with Cellomics Arrayscan to observe the cell growth with GFP signal. Consequently, the statistical analysis of the data collected was performed to create a growth curve for the six days. Each experiment was performed in triplicate.

MTT assay. The effects of AURKA silence on proliferation of A549 cells were analyzed by MTT (3-(4,5-dimethylthiazol2-yl)-2,5-diphenyltetrazolium bromide) (Sigma Chemical Co.). The A549 cells were plated at a final concentration of
Table I. Distribution of Aurora-A status in lung adenocarcinoma according to clinicopathological characteristics.

\begin{tabular}{lcccc}
\hline Characteristics & $\begin{array}{c}\text { Number of } \\
\text { patients }\end{array}$ & High & Low & p-value \\
\hline Gender & & & & \\
$\quad$ Male & 56 & 28 & 28 & 0.742 \\
Female & 45 & 24 & 21 & \\
Age (years) & & & & \\
$<60$ & 41 & 17 & 24 & 0.317 \\
$\geq 60$ & 60 & 30 & 30 & \\
Differentiation & & & & \\
Well & 24 & 9 & 13 & 0.122 \\
Moderate-poor & 77 & 43 & 34 & \\
Nodal status & & & & \\
N0 & 46 & 24 & 22 & 0.956 \\
N1 & 55 & 29 & 26 & \\
\hline
\end{tabular}

$5 \times 10^{3}$ cells/well in 96-well culture plates for different culture times. MTT $(10 \mu \mathrm{l})(5 \mathrm{mg} / \mathrm{ml}$ in PBS$)$ was added to each well and incubated for an additional $3 \mathrm{~h}$ at $37^{\circ} \mathrm{C}$. The formazan crystals were dissolved in $100 \mu \mathrm{l}$ of DMSO, and the absorbance was read at $490 \mathrm{~nm}$ by an ELISA reader (ELx808, Bio-Tek Instruments, Winooski, VT, USA).

Colony-formation assay. H1299 cells in all experimental groups were trypsinized and resuspended in complete medium. Two groups (scr-siRNA, AURKA-siRNA) of H1299 cells were plated in 96-well plates at the rate of 500 cells per perforation. Three compound perforations were set in each experimental group. The medium was changed and cells were monitored every 3 days. After 2 weeks of culture, the cells were washed with PBS. These perforations were scanned and photographed with Cellomics ArrayScan, the number and size of clones within the perforations were analyzed.

Flow cytometry analysis. The infected cells were synchronized by exposure to serum-free medium for $24 \mathrm{~h}$ to induce starvation. Then adherent cells were harvested by trypsinization, washed twice with ice-cold PBS, fixed in $70 \%$ ethanol and incubated for $30 \mathrm{~min}$ at $4^{\circ} \mathrm{C}$. After the ethanol was discarded by centrifugation, the fixed cells suspended in PI/RNase/ PBS (100 $\mu \mathrm{g} / \mathrm{ml}$ propidium iodide and $10 \mu \mathrm{g} / \mathrm{ml}$ RNase A) for $45 \mathrm{~min}$ at room temperature in the dark. After the suspension was filtered through a 50- $\mu$ m nylon mesh, the DNA content of the stained nuclei was analyzed by a flow cytometer to determine the percentage of cells for each phase of the cell cycle. Each experiment was performed in triplicate.

A total of $1.0 \times 10^{6}$ cells were collected and washed with ice-cold PBS for twice. Cells were resuspended in $100 \mu \mathrm{l}$ of Annexin $\mathrm{V}$ binding buffer, incubated with APC labeled Annexin $\mathrm{V}$ at room temperature for $15 \mathrm{~min}$. Apoptotic cells were detected by a flow cytometer. Each experiment was performed in triplicate. 

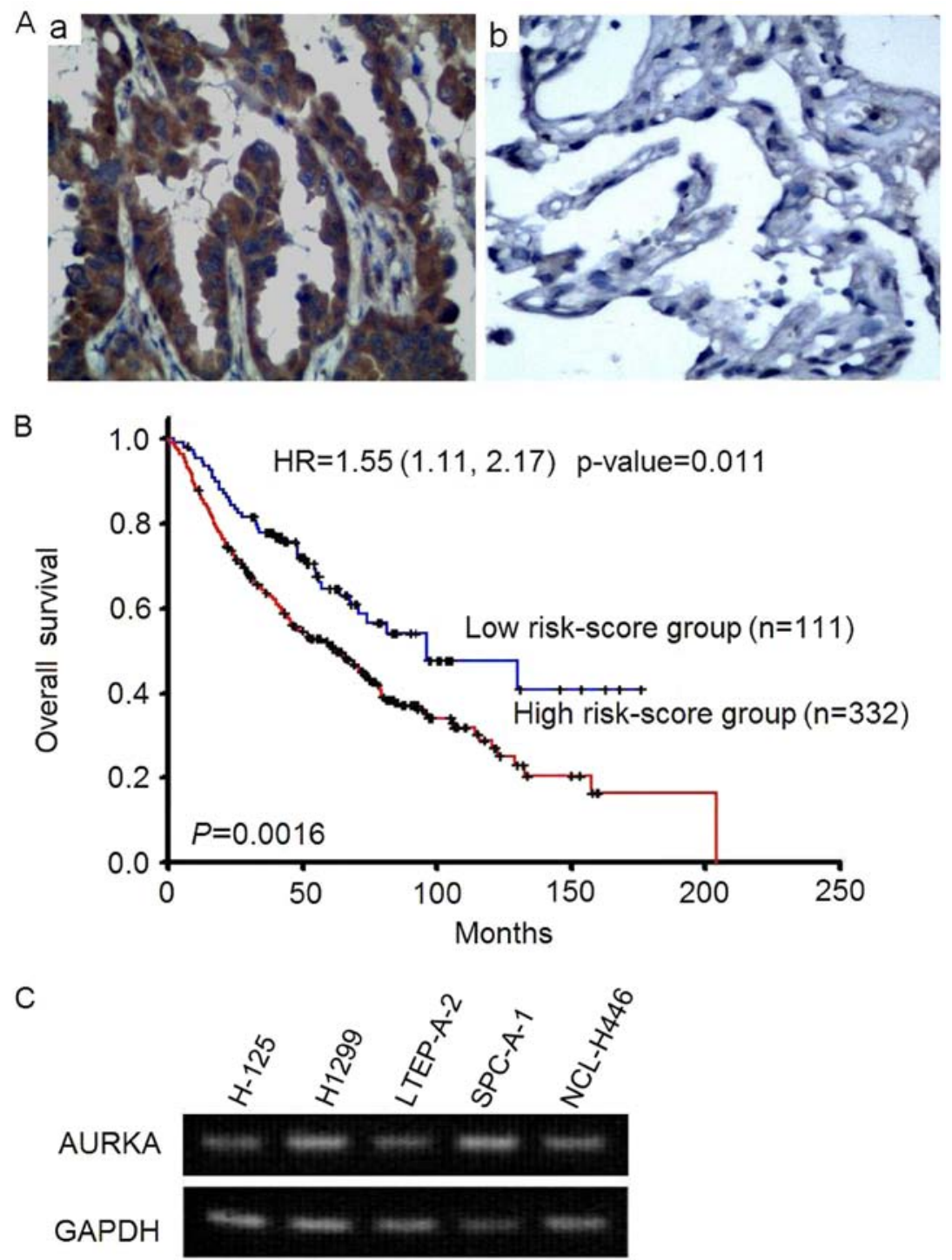

Figure 1. Analysis for expression of AURKA. (A) Expressions of AURKA protein in normal and lung adenocarcinoma tissue. (a) Lung adenocarcinoma (x400); (b) normal tissue (x400). (B) Relationship between AURKA expression and overall survival in lung adenocarcinoma by Kaplan-Meier analysis; (C) RT-PCR analysis for AURKA mRNA levels in NSCLC cells.

Western blot analysis. The cell pellets were lysed in lysis buffer that was supplemented with protease and phosphatase inhibitor cocktails. Total protein $(50 \mu \mathrm{g})$ was separated by SDS-PAGE and electroblotted onto nitrocellulose membranes after protein quantitation using Coomassie brilliant blue assay. Membranes were blocked by $5 \%$ non-fat dry milk and incubated for $1 \mathrm{~h}$ with mouse monoclonal antibodies against Aurora-A, WEE1, CDK4, EGFR, PAK4, RAF-1, CCND2, CCND3. After incubation with the secondary antibodies (peroxidase-conjugated anti-mouse $\operatorname{IgG}$ ) for $1 \mathrm{~h}$, protein bands were visualized by enhanced chemiluminescence. GAPDH was used as an internal positive control.

BrdU incorporation assay. The A549 cells infected with control lentivirus or AURKA-siRNA lentivirus were cultured for $72 \mathrm{~h}$ at $37^{\circ} \mathrm{C}$ in a humidified incubator with $5 \% \mathrm{CO}_{2}$. Then cells were trypsinized, resuspended, spread onto 96 -well plates and VCR was added after $12 \mathrm{~h}$. The treated cells were cultured for 24 and $96 \mathrm{~h}$, respectively, and incubated with BrdU for $4 \mathrm{~h}$. Subsequently, the cells were fixed, washed and incubated with mouse anti-BrdU antibody for $1 \mathrm{~h}$ and horseradish peroxidase-conjugated secondary antibodies for $30 \mathrm{~min}$ following the manufacturer's protocol (Chemicon International Inc., Temecula, CA, USA). The immune complexes were detected by the subsequent 3,3',5,5'-Tetramethyl-benzidine (TMB) substrate reaction, and the levels of BrdU incorporated into cells were quantified by measuring the absorbance at $490 \mathrm{~nm}$ using a microplate reader (Bio-Rad 680, Bio-Rad, Hercules, CA, USA).

Chemotherapy and apoptosis assay. To investigate whether the transfection with lentivirus encoding a AURKA siRNA increases the chemosensitivity of lung adenocarcinoma cells, A549 cells were treated with VCR at 50, $100 \mathrm{nM}$ for $48 \mathrm{~h}$ after transfection with AURKA-siRNA and scr-siRNA, respectively.

Then A549 cells in each group were harvested and cells stained with the Annexin V apoptosis kit (Invitrogen) according to the manufacturer's instructions. Analysis of apoptosis were performed using a FACScan flow cytometer (Becton-Dickinson). 
A

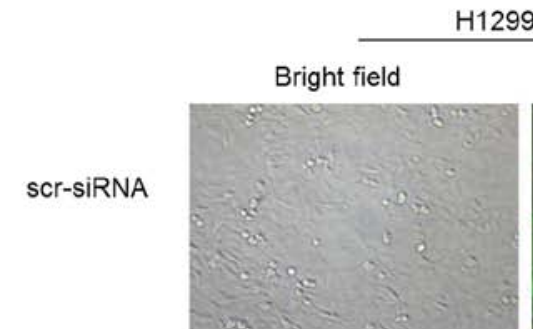

1299 cells
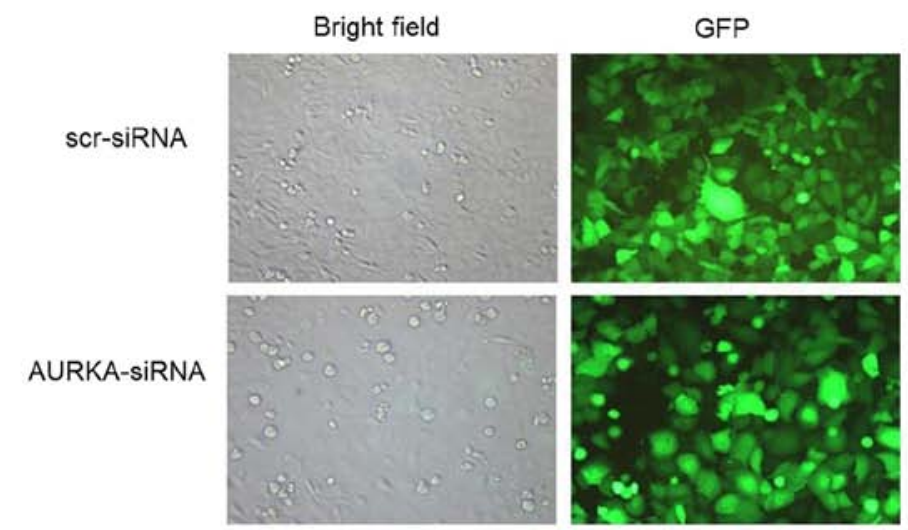

B

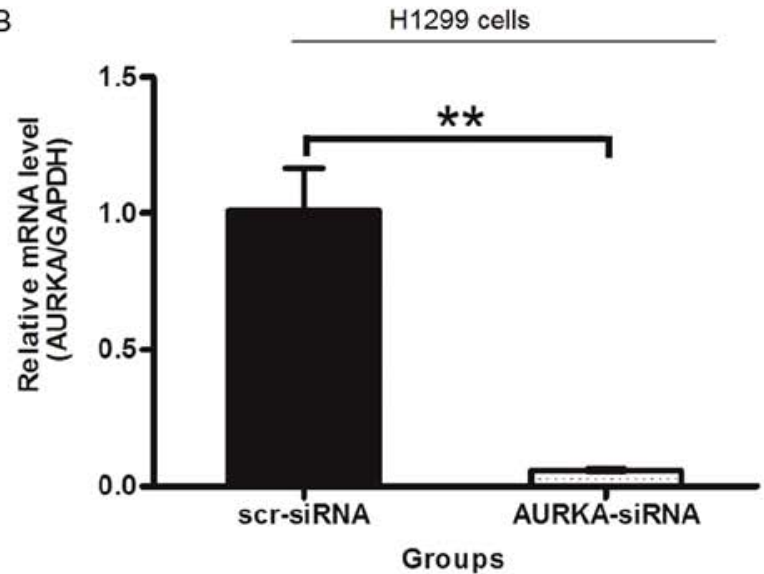

C

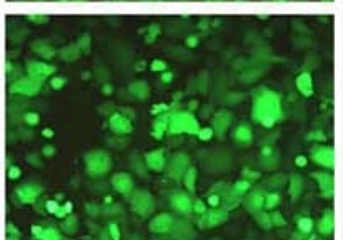

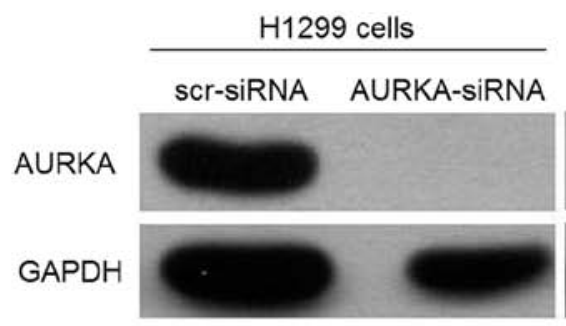

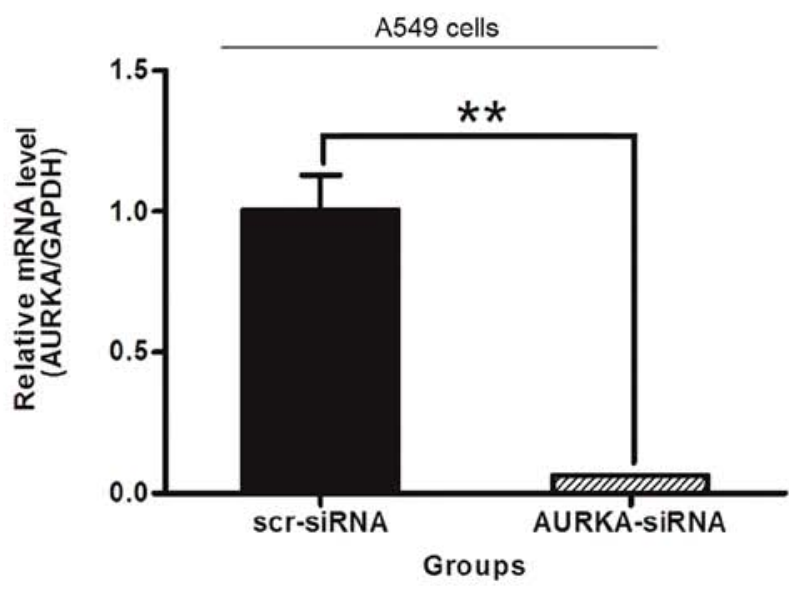

A549 cells
A549 cells GFP
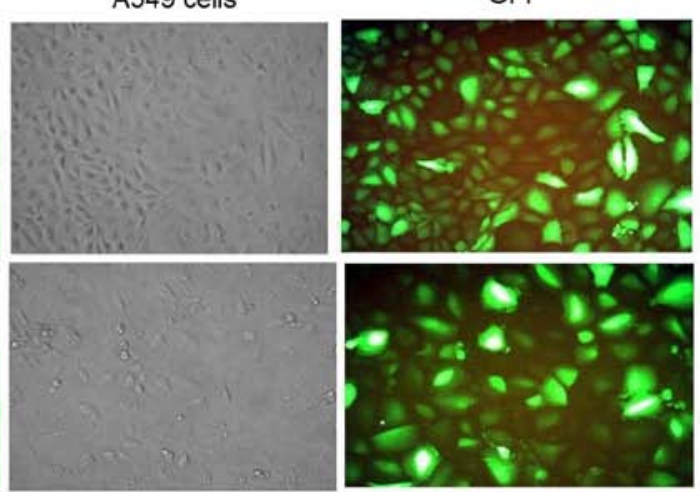

(n) 
A
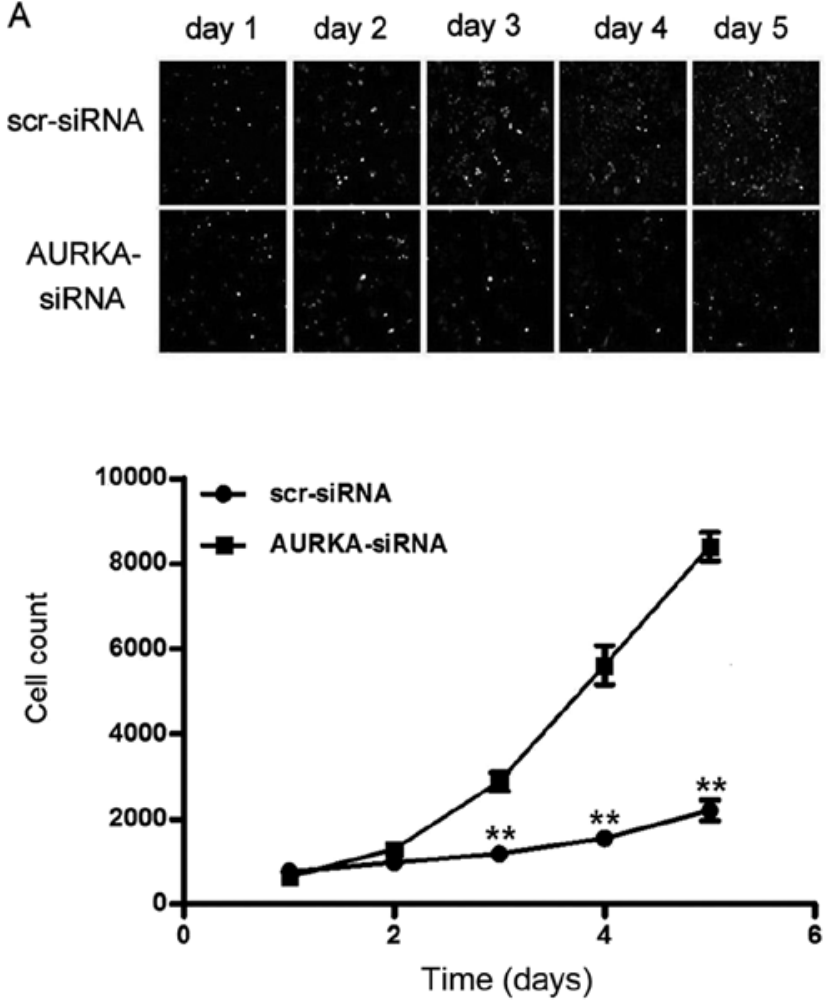

B
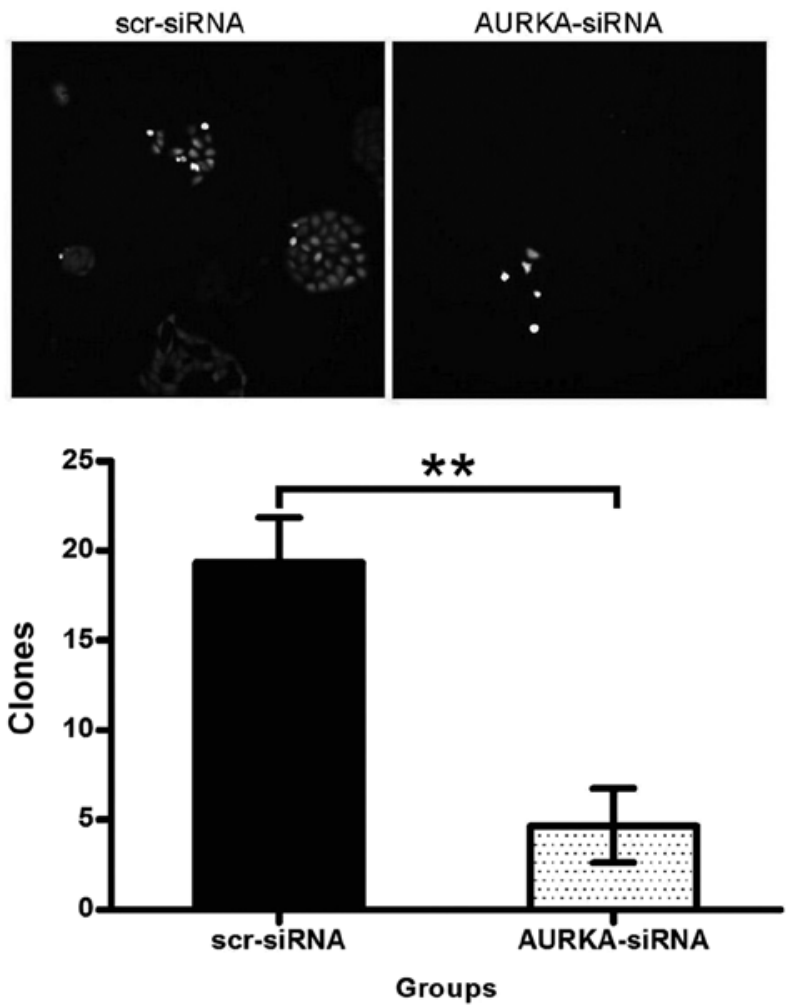

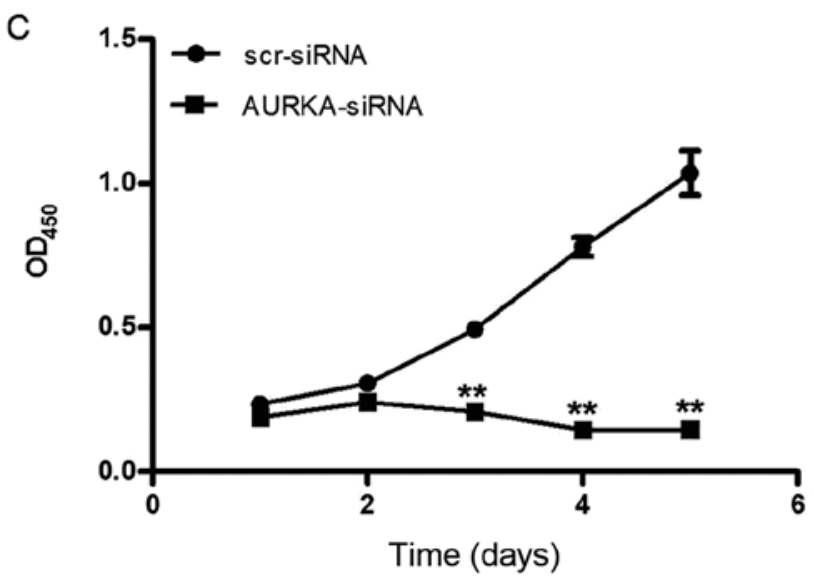

Figure 3. Effect of AURKA silencing on the proliferation of lung adenocarcinoma cells. (A) Cellomics arrayscan assay. AURKA-siRNA depressed the growth curves of H1299 cells as compared with the scr-siRNA as determined using a Cellomics arrayscan with time-dependent relation. (B) Colony formation assay. Colony formation assay showed that AURKA siRNA decreased the clone numbers of H1299 cells as compared with the scr-siRNA group. (C) MTT assay. The proliferation levels were measured by MTT assay in AURKA-silenced and -non-silenced A549 cells. AURKA-siRNA depressed the growth curves of H1299 cells as compared with the scr-siRNA group. ${ }^{* *} \mathrm{p}<0.01$, in comparison with the scr-siRNA group.

lines H-125, H1299, LTEP-A-2, SPC-A-1 and human small cell lung cancer NCL-H446 cells. These results suggests a correlation between the overexpression of AURKA and the occurrence of NSCLC.

Lentivirus-mediated RNAi can efficiently block expression of AURKA. To further illuminate the role of AURKA in lung adenocarcinoma, we constructed the lentivirus-delivered AURKA-specific siRNA vector (AURKA-siRNA) and scramble-siRNA vector (scr-siRNA). Fluorescent microscope was used to investigate the lentiviral infection efficiency. The results showed that $>90 \%$ of the cells exhibited the green fluorescence indicative of infection after the transfection (Fig. 2A). To determine the silencing efficiency, the expression levels of
AURKA mRNA and protein were detected by real-time PCR and western blotting. The results indicated that the levels of RNA (Fig. 2B) and protein (Fig. 2C) expression of AURKA were dramatically decreased in both H1299 and A549 cells compared to scr-siRNA treatment groups. Thus, these results confirmed that the AURKA-siRNA could downregulate the AURKA expression effectively.

Inhibition of cell growth of lung adenocarcinoma cells by depletion of AURKA. We tested the effect of AURKAsiRNA on the cell viability of H1299 and A549 cells in vitro. Cellomics analysis showed that AURKA knockdown significantly inhibited cell growth of H1299 cells comparing with scr-siRNA treatment, and the difference 


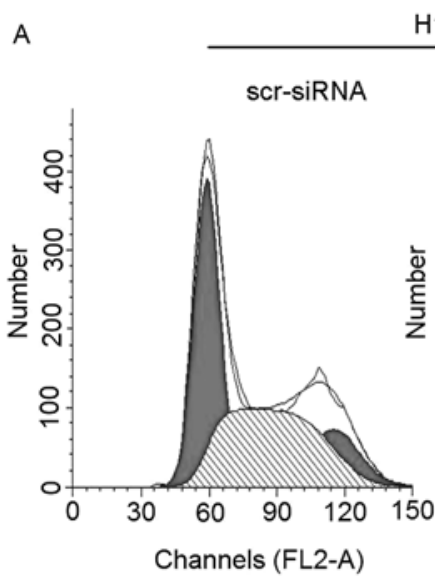

H1299 cells
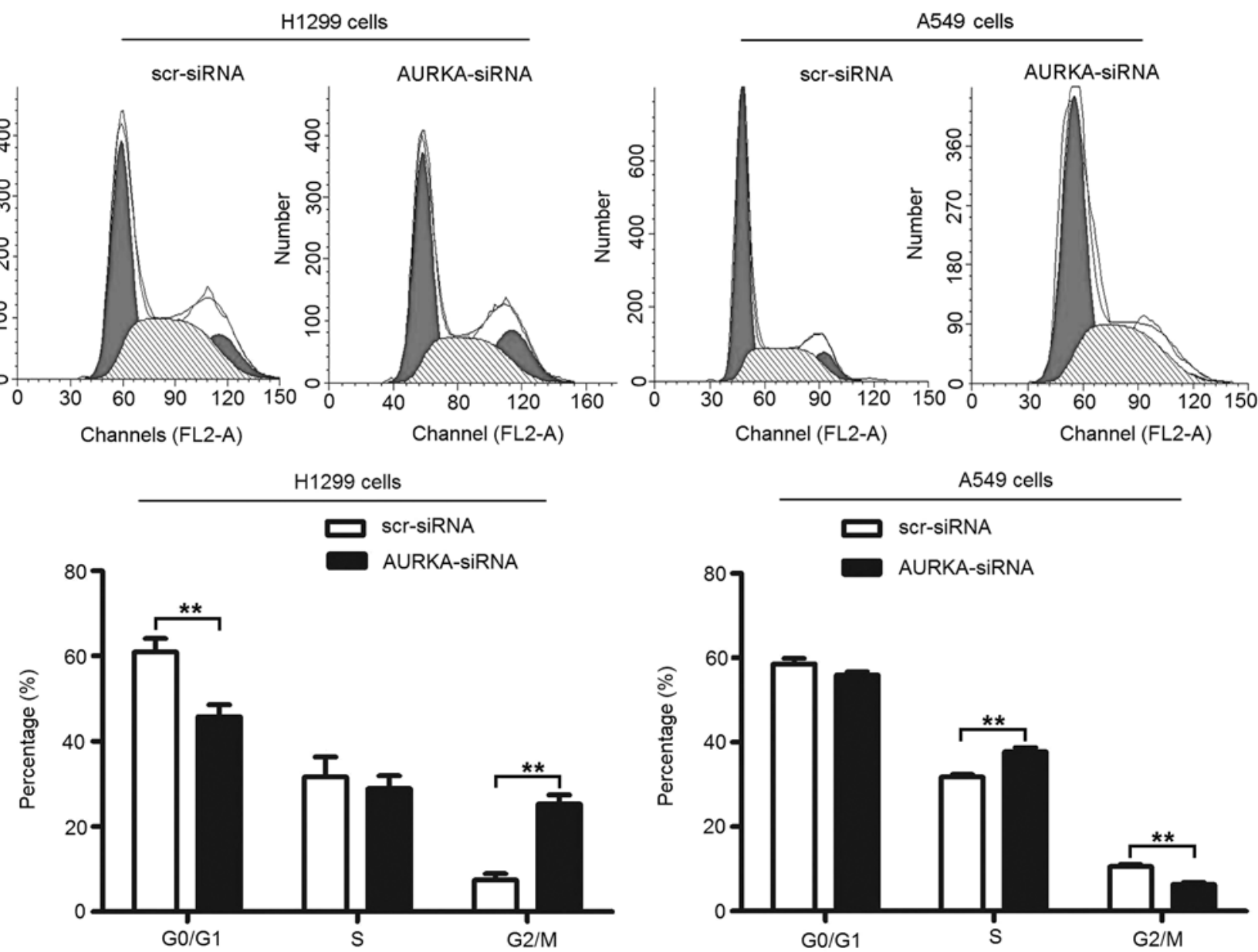

Figure 4. The effects of AURKA siRNA on the cell cycle distribution of lung adenocarcinoma cells as determined by flow cytometric analysis. (A) The flow cytometric histogram and the number of cells under each phase of the cell cycle in AURKA-silenced and -non-silenced H1299 and A549 cells. (B) The numerical representation of the cells under each phase of the cell cycle in AURKA-silenced and -non-silenced H1299 and A549 cells.

was more pronounced with time-dependent manner $(\mathrm{p}<0.01)$ (Fig. 3A). The results of the colony formation assay show that the number of colonies in the AURKA-siRNA group (4.67 \pm 2.08$)$ was significantly less than that in the scr-siRNA group $(19.33 \pm 2.52)$ in H1299 cells $(\mathrm{p}<0.01)$ (Fig. 3B). These results demonstrate that the reduction in AURKA expression decreases the ability of H1299 cells to form colonies.

MTT assay was performed to study the effect of AURKAsiRNA on A549 cell growth. As shown in Fig. 3C, A549 cells show a significant $(\mathrm{p}<0.01)$ reduction in cell viability 5 days after infection. The results suggests that silencing of AURKA gene inhibits the proliferation of lung adenocarcinoma cells.

Knockdown of AURKA leads to alterations in cell cycle of lung adenocarcinoma cells. In order to study the mechanisms underlying RNAi-mediated proliferation inhibition, the changes in the cell cycle was detected by flow cytometric analysis of the DNA content. As shown in Fig. 4A and B, treatment with AURKA-siRNA results in an increase in the percentage of $\mathrm{H} 1299$ cells in the G2/M phase from $7.40 \pm 1.57 \%$ to $25.28 \pm 2.18 \%(\mathrm{p}<0.01)$. In accordance with this increase in the percentage of cells in the $\mathrm{G} 2 / \mathrm{M}$ phase, there was a significant decrease in the percentage of cells in the G0/G1 phase from $60.94 \pm 3.14 \%$ to $45.82 \pm 2.75 \%$ $(\mathrm{p}<0.01)$, but no significant change in the percentage of cells in the $\mathrm{S}$ phase from $31.66 \pm 4.69 \%$ to $28.90 \pm 3.04 \%(\mathrm{p}>0.05)$. Treatment with AURKA-siRNA also results in an decrease in the percentage of A549 cells in the G2/M phase from $10.57 \pm 0.53 \%$ to $6.27 \pm 0.57 \%$ ( $p<0.01)$. Again, there was also a significant increase in the percentage of cells in the $S$ phase from $31.77 \pm 0.61 \%$ to $37.74 \pm 1.01 \%$ ( $\mathrm{p}<0.01$ ), but no significant change in the percentage of cells in the G0/G1 phase from $58.63 \pm 1.30 \%$ to $55.99 \pm 0.67 \%$ ( $\mathrm{p}>0.05)$.

These results suggest that depletion of AURKA inhibits the cellular proliferation of lung adenocarcinoma cells via G2/M and S phase arrest of the cell cycle in H1299 and A549 cells, respectively. The inconsistent results of cell cycling may derive from the endogenous differences in cell cycle in different cell types.

Induction of apoptosis in lung adenocarcinoma cells by AURKA knockdown. To determine whether knockdown of AURKA could induce cell apoptosis, flow cytometry was used to analyze the apoptosis of lung adenocarcinoma cells after infection with AURKA-siRNA for $72 \mathrm{~h}$. As shown in Fig. 5A and B, the percentage of apoptotic H1299 cells was $4.14 \pm 0.46 \%$ in scr-siRNA group and the percentage of apoptotic cells increased to $18.04 \pm 2.69 \%$ in AURKA-siRNA 

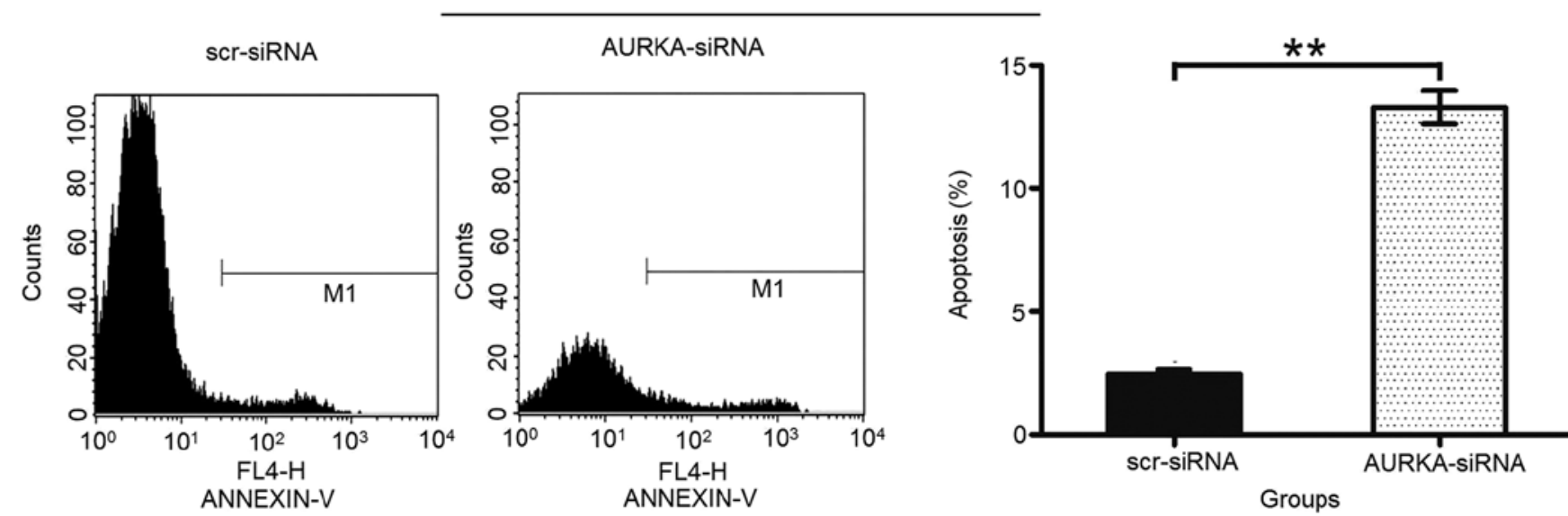

B
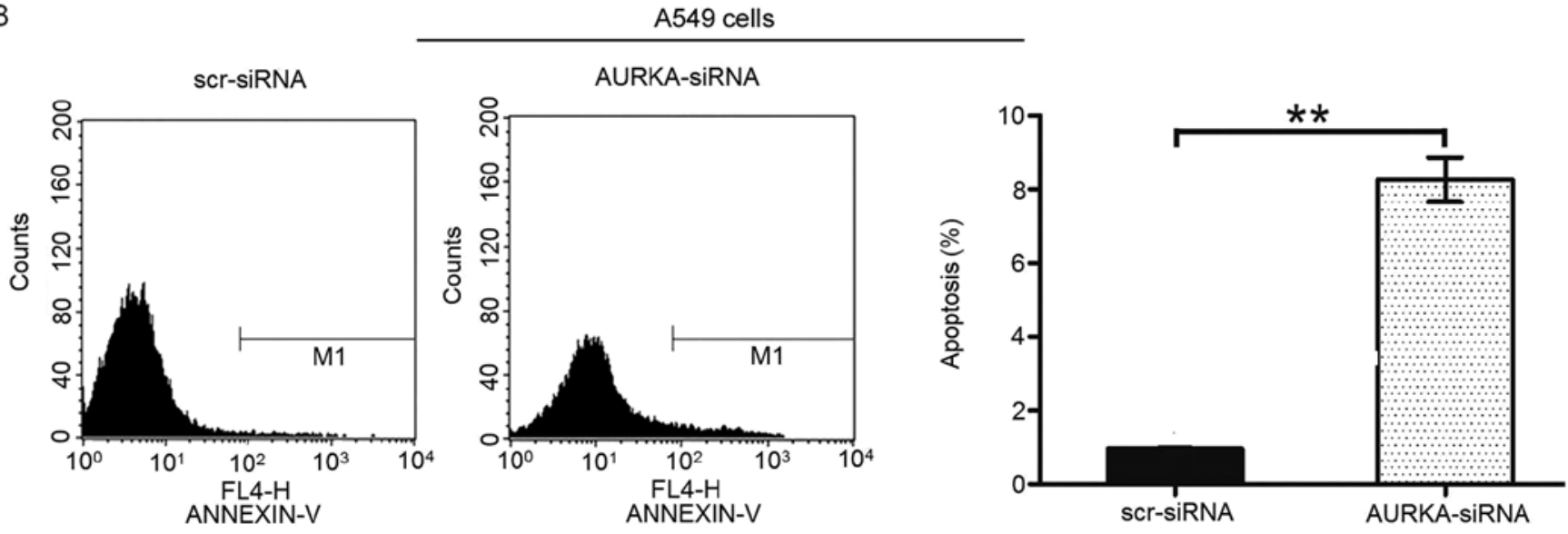

Figure 5. The effects of AURKA siRNA on the cell apoptosis of lung adenocarcinoma cells as determined by flow cytometric analysis. (A) Flow cytometry analysis shows that knockdown of AURKA expression via AURKA siRNA increased cell apoptosis in H1299 cells. (B) Flow cytometry analysis showed that knockdown of AURKA expression via AURKA siRNA increased cell apoptosis in A549 cells. ${ }^{* *}$ p $<0.01$, in comparison with scr-diRNA group.

group (p<0.01) (Fig. 5A). The percentage of apoptotic A549 cells was $0.98 \pm 0.02 \%$ in scr-siRNA group cells and increased to $8.27 \pm 0.61 \%$ in AURKA-siRNA group (p<0.01) (Fig. 5B). These data suggest that the depletion of AURKA specifically induced apoptosis of the lung adenocarcinoma cells.

Suppression of AURKA alters the expression levels of cell cycle-related genes. To test the possible mechanisms underlying the lung adenocarcinoma cell proliferation inhibition and apoptosis after AURKA knockdown, we checked cell cycle-related gene expression. Our results reveals that the knockdown of AURKA downregulated RAF-1, CCND2, CCND3, CDK4, PAK4, EGFR and upregulated WEE1 expression in H1299 cells. These results indicate that the heightened apoptosis associated with AURKA downregulation may be partly mediated by cell cycle-related proteins in H1299 cells (Fig. 6A).

Knockdown of AURKA enhances chemotherapy sensitivity to $V C R$ in human lung carcinoma A549 cells. The cooperative effects of AURKA knockdown and VCR on repressing lung adenocarcinoma cell proliferation were investigated in this study. AURKA knockdown cooperated with VCR to inhibit A549 cell proliferation. As showed in Fig. 6B, at both low
(50 nM) and high concentration (100 nM) of VCR, AURKA depletion could cooperatively inhibit A549 cell growth in vitro.

The number of apoptotic cells was determined by Annexin V staining. A549 cells were transfected with scr-siRNA or AURKA-siRNA, and then treated with VCR for 48 h. As shown in Fig. 6C, the percentage of apoptotic A549 cells was $9.79 \pm 0.18 \%$ in scr-siRNA + VCR (50 nM) group and the percentage of apoptotic cells increased to $38.81 \pm 0.88 \%$ in AURKA-siRNA + VCR $(50 \mathrm{nM})$ group $(\mathrm{p}<0.01)$. The percentage of apoptotic A549 cells was $16.30 \pm 0.11 \%$ in scr-siRNA + VCR (100 nM) group cells and increased to $48.58 \pm 0.63 \%$ in AURKA-siRNA + VCR $(100 \mathrm{nM})$ group $(\mathrm{p}<0.01)$. These data suggest that the depletion of AURKA cooperatively induces apoptosis of the lung adenocarcinoma cells.

\section{Discussion}

AURKA functions as an oncogene in several malignancies and its overexpression is associated with a higher grade of tumor and a poor prognosis. Aneuploidy is associated with a poor outcome and a marker of metastasis in gastric carcinoma, a correlation between aneuploidy and AURKA overexpression exists in gastric cancer, clinical samples with gene amplification and overexpression of AURKA showed 

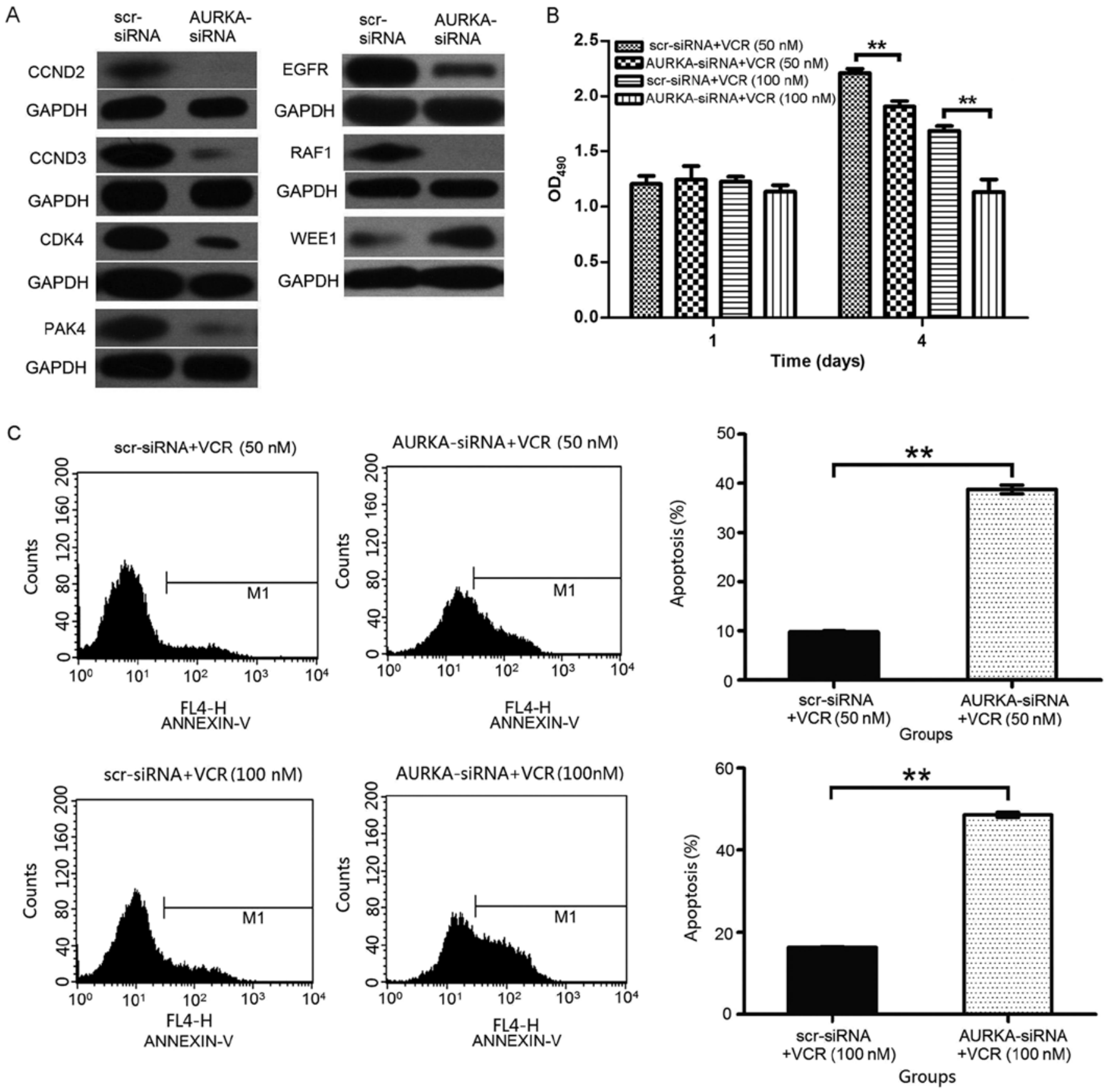

Figure 6. Western blotting, BrdU incorporation and flow cytometry analysis. (A) AURKA silencing downregulated RAF-1, CCND2, CCND3, CDK4, PAK4, EGFR and upregulated WEE1 expression in H1299 cells. (B) The DNA synthesis rate was analyzed by BrdU incorporation assay on the 1st and 4th days. Downregulated AURKA in human lung cancer A549 cells displayed changes in DNA synthesis. Silencing AURKA gene expression by siRNA can significantly enhance chemotherapy sensitivity effect by combining VCR in lung cancer A549 cells. (C) Apoptosis sensitivity of A549 cells towards VCR after downregulation of AURKA. The rate of apoptosis of A549 cells was significantly higher in A549 cells were treated with VCR after transfection with AURKAsiRNA than those treated with VCR after transfection with scr-siRNA. ${ }^{* *} \mathrm{p}<0.01$, in comparison with control.

aneuploidy and poor prognosis $(7,14)$. In this study, we also observed that AURKA is highly expressed in lung adenocarcinoma tissue and its overexpression is associated with shorter overall survival, which indicates that AURKA plays important roles in the development of lung adenocarcinoma.

AURKA promotes cell cycle progression by regulating important mitotic events including spindle assembly, chromosome maturation and mitotic entry $(9,10)$. Depletion of AURKA caused cell cycle arrest in G2/M and S phases in H1229 and A549 cells, respectively. In addition, knockdown of
AURKA in such cells could induce apoptosis. Our results are similar as other studies, which suggest that AURKA inhibition is potential therapeutic for lung adenocarcinoma. This is first study that ties AURKA with lung adenocarcinoma in tumor development and treatment.

To elucidate the mechanisms of cell cycle arrest and apoptosis after knockdown of AURKA, we checked the expression of several cell cycle related genes. D-type cyclins (D1, D2, and D3) are a family of key cell cycle regulators, as they can promote cell cycle progression by binding to and acti- 
vating cyclin-dependent kinase $4(\mathrm{cdk} 4) / \mathrm{cdk} 6$. The activated cyclin D-cdk4/cdk6 complex can then phosphorylate and deactivate the tumor suppressor protein $\mathrm{pRB}$, this phosphorylation in turn leads to the release and upregulation of transcription factor E2F that promote progression from the $\mathrm{G} 1$ to $\mathrm{S}$ phase of the cell cycle $(15,16)$. Aberrant expression of CCND2 can lead to unrestricted cell proliferation. Its aberrant expression has been observed in various cancers. Many studies have found that CCND2 is overexpressed or amplified in many human cancers, such as $\mathrm{CaP}$ prostate cancer, gastric cancer, ovarian and testicular tumors (17-19). Cyclin D3 has been suggested to have a role in certain cancers. Moreover, overexpression of cyclin D3 has been found in several human cancers, such as renal cell carcinoma, pancreatic adenocarcinoma and breast carcinoma (20-22).

CDK4 belongs to the cyclin dependent kinases family, it has been found to promote cell proliferation by driving cell cycle progression (23-25). Overexpression of CDK4 protein has been described in many tumors, including oral squamous cell carcinoma, pancreatic neuroendocrine tumor (NET), and lung cancer (26-28). Patients with lung cancer with higher CDK4 expression levels had a markedly shorter overall survival time than those with low CDK4 expression (28).

PAK4 (P21-activated kinase 4), a subfamily of serine/ threonine protein kinases involved in cytoskeletal dynamics and cell motility, plays a crucial role in oncogenic signaling pathways. PAK4 is thought to regulate cancer cell progression involving the c-Src/EGFR/cyclin D1 pathway $(29,30)$. PAK4 upregulation has been identified in many kinds of human cancer cell lines and amplification of the chromosome region containing PAK4 has been frequently observed in colorectal, pancreatic, and ovarian cancer (31-34).

Epidermal growth factor receptor (EGFR), a receptor tyrosine kinase (TK), is the expression product of oncogene c-erbB1 and plays essential roles in cell differentiation, proliferation, development and maintenance in both cancerous and normal physiological conditions. Expression of EGFR strongly affects the outcomes of cancer patients in many cancer types. It has been found to act as a powerful indicator with tumor progression and poor survival $(35,36)$. EGFR is frequently aberrantly activated in NSCLC (37-39).

V-raf-1 murine leukemia viral oncogene homolog 1 (Raf-1) is a multifunctional protein with serine and threonine kinase activity. It is a critical target of many growth factors in various cell types. Raf-1 is at the apex of the mitogen activated protein kinase (MEK)-ERK pathway, which controls a variety of fundamental cellular including cell proliferation, survival and migration including cell proliferation, migration, survival, and transformation (40-42). In the present study, we found that the knockdown of AURKA downregulated RAF-1, CCND2, CCND3, CDK4, PAK4, EGFR, which indicates the potential mechanisms of AURKA depletion-induced cell cycle arrest and apoptosis in NSCLC cell lines.

WEE1, a tyrosine kinase regulator of the cell cycle, has been associated with survival in several cancer types, including malignant melanoma, breast cancer and glioblastoma. WEE1 was reported to be a safeguard against mitotic catastrophe in instances of sensitive cell division. Its overexpression causes G2 arrest by promoting the inhibitory phosphorylation of cyclin-dependent kinase (43-45). Our results show that upregulated expression of WEE1 in H1299 cells after AURKA knockdown, suggesting other mechanisms of AURKA inhibition in treatment of lung adenocarcinoma.

VCR has been extensively used in clinic and its anticancer mechanisms are through acting on tubulin, inhibiting the cell mitosis and arresting cell cycling and proliferation, which are similar with those of AURKA depletion. Therefore, we speculats that silencing of AURKA gene may enhance VCR sensitivity and reduce drug resistance. To further confirm that AURKA inhibition has cooperative effects on repressing lung adenocarcinoma cell proliferation, we treated A549 cells with different doses of VCR combined with AURKA knockdown. The results are very interesting; the AURKA knockdown enhanced the repressing effects of VCR on A549 cell proliferation. This is direct evidence that AURKA depletion could combine with traditional chemotherapy drug for treating lung adenocarcinoma.

In summary, this study firstly demonstrats that AURKA is a therapeutic target for treatment of lung adenocarcinoma. AURKA depletion could induce cell cycle arrest and apoptosis in lung adenocarcinoma cells. Cooperative effects with VCR provided direct evidence that AURKA is a target for lung adenocarcinoma therapy. The detailed mechanisms should be elucidated and clinical trials performed in the future.

\section{Acknowledgements}

This study was supported by a grant from the Natural Science Foundation for the Youth (no. 81402220), Suzhou Planning Project of Science and Technology (SYS201301) and the Science and Technology Foundation of Kunshan City (no. ks1234). The authors thank Dr Wenxiang Wei (Soochow University, Suzhou 215123, China) for his sincere help and technical support.

\section{References}

1. Siegel R, Naishadham D and Jemal A: Cancer statistics, 2013. CA Cancer J Clin 63: 11-30, 2013.

2. Chou HC and Chan HL: Effect of glutathione reductase knockdown in response to UVB-induced oxidative stress in human lung adenocarcinoma. Proteome Sci 12: 2, 2014.

3. Pilotto S, Bria E, Peretti U, Massari F, Garassino M, Pelosi G and Tortora G: Lung adenocarcinoma patient refractory to gefitinib and responsive to crizotinib, with concurrent rare mutation of the epidermal growth factor receptor (L861Q) and increased ALK/ MET/ROS1 gene copy number. J Thorac Oncol 8: e105-e106, 2013.

4. Onn A, Tsuboi M and Thatcher N: Treatment of non-small-cell lung cancer: A perspective on the recent advances and the experience with gefitinib. Br J Cancer 91 (Suppl 2): S11-S17, 2004.

5. Fang W, Zhang J, Liang W, Huang Y, Yan Y, Wu X, Hu Z, Ma Y, Zhao $\mathrm{H}$, Zhao Y, et al: Efficacy of epidermal growth factor receptor-tyrosine kinase inhibitors for Chinese patients with squamous cell carcinoma of lung harboring EGFR mutation. J Thorac Dis 5: 585-592, 2013.

6. Shimada Y, Saji H, Nomura M, Matsubayashi J, Yoshida K, Kakihana M, Kajiwara N, Ohira T and Ikeda N: Cancer stem cell-related marker expression in lung adenocarcinoma and relevance of histologic subtypes based on IASLC/ATS/ERS classification. Onco Targets Ther 6: 1597-1604, 2013.

7. Dar AA, Goff LW, Majid S, Berlin J and El-Rifai W: Aurora kinase inhibitors - rising stars in cancer therapeutics? Mol Cancer Ther 9: 268-278, 2010. 
8. Kallioniemi A, Kallioniemi OP, Piper J, Tanner M, Stokke T, Chen L, Smith HS, Pinkel D, Gray JW and Waldman FM: Detection and mapping of amplified DNA sequences in breast cancer by comparative genomic hybridization. Proc Natl Acad Sci USA 91: 2156-2160, 1994.

9. Romain C, Paul P, Kim KW, Lee S, Qiao J and Chung DH: Targeting Aurora kinase-A downregulates cell proliferation and angiogenesis in neuroblastoma. J Pediatr Surg 49: 159-165, 2014

10. Thrane S, Pedersen AM, Thomsen MB, Kirkegaard T, Rasmussen BB, Duun-Henriksen AK, Lænkholm AV, Bak M, Lykkesfeldt AE and Yde CW: A kinase inhibitor screen identifies Mcl-1 and Aurora kinase A as novel treatment targets in antiestrogen-resistant breast cancer cells. Oncogene 34: 4199-4210, 2015.

11. Elbashir SM, Harborth J, Lendeckel W, Yalcin A, Weber K and Tuschl T: Duplexes of 21-nucleotide RNAs mediate RNA interference in cultured mammalian cells. Nature 411: 494-498, 2001

12. Shedden K, Taylor JM, Enkemann SA, Tsao MS, Yeatman TJ, Gerald WL, Eschrich S, Jurisica I, Giordano TJ, Misek DE, et al; Director's Challenge Consortium for the Molecular Classification of Lung Adenocarcinoma: Gene expression-based survival prediction in lung adenocarcinoma: A multi-site, blinded validation study. Nat Med 14: 822-827, 2008.

13. Zielske SP and Stevenson M: Importin 7 may be dispensable for human immunodeficiency virus type 1 and simian immunodeficiency virus infection of primary macrophages. J Virol 79: 11541-11546, 2005.

14. Jeng YM, Peng SY, Lin CY and Hsu HC: Overexpression and amplification of Aurora-A in hepatocellular carcinoma. Clin Cancer Res 10: 2065-2071, 2004.

15. Sherr CJ: G1 phase progression: Cycling on cue. Cell 79: 551-555, 1994.

16. Malumbres $M$ and Barbacid $M$ : Cell cycle, CDKs and cancer: $A$ changing paradigm. Nat Rev Cancer 9: 153-166, 2009.

17. Dong Q, Meng P, Wang T, Qin W, Qin W, Wang F, Yuan J, Chen Z, Yang A and Wang H: MicroRNA let-7a inhibits proliferation of human prostate cancer cells in vitro and in vivo by targeting E2F2 and CCND2. PLoS One 5: e10147, 2010.

18. Mermelshtein A, Gerson A, Walfisch S, Delgado B, Shechter-Maor G, Delgado J, Fich A and Gheber L: Expression of D-type cyclins in colon cancer and in cell lines from colon carcinomas. Br J Cancer 93: 338-345, 2005.

19. Susaki E, Nakayama K and Nakayama KI: Cyclin D2 translocates p27 out of the nucleus and promotes its degradation at the G0-G1 transition. Mol Cell Biol 27: 4626-4640, 2007.

20. Hedberg Y, Roos G, Ljungberg B and Landberg G: Cyclin D3 protein content in human renal cell carcinoma in relation to cyclin D1 and clinico-pathological parameters. Acta Oncol 41: 175-181, 2002.

21. Ito Y, Takeda T, Wakasa $K$, Tsujimoto $M$ and Matsuura N: Expression and possible role of cyclin D3 in human pancreatic adenocarcinoma. Anticancer Res 21: 1043-1048, 2001.

22. Wong SC, Chan JK, Lee KC and Hsiao WL: Differential expression of p16/p21/p27 and cyclin D1/D3, and their relationships to cell proliferation, apoptosis, and tumour progression in invasive ductal carcinoma of the breast. J Pathol 194: 35-42, 2001.

23. Retzer-Lidl M, Schmid RM and Schneider G: Inhibition of CDK4 impairs proliferation of pancreatic cancer cells and sensitizes towards TRAIL-induced apoptosis via downregulation of survivin. Int J Cancer 121: 66-75, 2007.

24. Karim BO, Rhee KJ, Liu G, Zheng D and Huso DL: Chemoprevention utility of silibinin and $\mathrm{Cdk} 4$ pathway inhibition in Apc(-/+) mice. BMC Cancer 13: 157, 2013.

25. Chan KC, Ting CM, Chan PS, Lo MC, Lo KW, Curry JE, Smyth T, Lee AW, Ng WT, Tsao GS, et al: A novel Hsp90 inhibitor AT13387 induces senescence in EBV-positive nasopharyngeal carcinoma cells and suppresses tumor formation. Mol Cancer 12: 128, 2013.

26. Poomsawat S, Buajeeb W, Khovidhunkit SO and Punyasingh J: Alteration in the expression of cdk4 and cdk6 proteins in oral cancer and premalignant lesions. J Oral Pathol Med 39: 793-799, 2010.
27. Tang LH, Contractor T, Clausen R, Klimstra DS, Du YC, Allen PJ, Brennan MF, Levine AJ and Harris CR: Attenuation of the retinoblastoma pathway in pancreatic neuroendocrine tumors due to increased cdk4/cdk6. Clin Cancer Res 18: 4612-4620, 2012.

28. Wu A, Wu B, Guo J, Luo W, Wu D, Yang H, Zhen Y, Yu X, Wang H, Zhou Y, et al: Elevated expression of CDK4 in lung cancer. J Transl Med 9: 38, 2011.

29. Sørensen CS and Syljuåsen RG: Safeguarding genome integrity: The checkpoint kinases ATR, CHK1 and WEE1 restrain CDK activity during normal DNA replication. Nucleic Acids Res 40: 477-486, 2012.

30. Callow MG, Clairvoyant F, Zhu S, Schryver B, Whyte DB, Bischoff JR, Jallal B and Smeal T: Requirement for PAK4 in the anchorage-independent growth of human cancer cell lines. J Biol Chem 277: 550-558, 2002 .

31. Siu MK, Chan HY, Kong DS, Wong ES, Wong OG, Ngan HY, Tam KF, Zhang H, Li Z, Chan QK, et al: p21-activated kinase 4 regulates ovarian cancer cell proliferation, migration, and invasion and contributes to poor prognosis in patients. Proc Natl Acad Sci USA 107: 18622-18627, 2010.

32. Zhang HJ, Siu MK, Yeung MC, Jiang LL, Mak VC, Ngan HY, Wong OG, Zhang HQ and Cheung AN: Overexpressed PAK4 promotes proliferation, migration and invasion of choriocarcinoma. Carcinogenesis 32: 765-771, 2011.

33. Liu Y, Xiao H, Tian Y, Nekrasova T, Hao X, Lee HJ, Suh N, Yang CS and Minden A: The pak4 protein kinase plays a key role in cell survival and tumorigenesis in athymic mice. Mol Cancer Res 6: 1215-1224, 2008.

34. Kimmelman AC, Hezel AF, Aguirre AJ, Zheng H, Paik JH, Ying H, Chu GC, Zhang JX, Sahin E, Yeo G, et al: Genomic alterations link Rho family of GTPases to the highly invasive phenotype of pancreas cancer. Proc Natl Acad Sci USA 105: 19372-19377, 2008.

35. Mitsudomi T and Yatabe Y: Epidermal growth factor receptor in relation to tumor development: EGFR gene and cancer. FEBS J 277: 301-308, 2010.

36. Cheng L, Zhang S, Alexander R, Yao Y, MacLennan GT, Pan CX, Huang J, Wang M, Montironi R and Lopez-Beltran A: The landscape of EGFR pathways and personalized management of non-small-cell lung cancer. Future Oncol 7: 519-541, 2011.

37. Pao W and Chmielecki J: Rational, biologically based treatment of EGFR-mutant non-small-cell lung cancer. Nat Rev Cancer 10: 760-774, 2010.

38. Yamamoto H, Toyooka S and Mitsudomi T: Impact of EGFR mutation analysis in non-small cell lung cancer. Lung Cancer 63: 315-321, 2009.

39. Inamura $\mathrm{K}$, Ninomiya $\mathrm{H}$, Ishikawa $\mathrm{Y}$ and Matsubara $\mathrm{O}$ : Is the epidermal growth factor receptor status in lung cancers reflected in clinicopathologic features? Arch Pathol Lab Med 134: 66-72, 2010.

40. Alejandro EU, Kalynyak TB, Taghizadeh F, Gwiazda KS, Rawstron EK, Jacob KJ and Johnson JD: Acute insulin signaling in pancreatic beta-cells is mediated by multiple Raf- 1 dependent pathways. Endocrinology 151: 502-512, 2010

41. Wang H, Gambosova K, Cooper ZA, Holloway MP, Kassai A, Izquierdo D, Cleveland K, Boney CM and Altura RA: EGF regulates survivin stability through the Raf-1/ERK pathway in insulin-secreting pancreatic $\beta$-cells. BMC Mol Biol 11: 66, 2010.

42. Yoon S and Seger R: The extracellular signal-regulated kinase: Multiple substrates regulate diverse cellular functions. Growth Factors 24: 21-44, 2006

43. Magnussen GI, Holm R, Emilsen E, Rosnes AK, Slipicevic A and Flørenes VA: High expression of Weel is associated with poor disease-free survival in malignant melanoma: Potential for targeted therapy. PLoS One 7: e38254, 2012.

44. Murrow LM, Garimella SV, Jones TL, Caplen NJ and Lipkowitz S: Identification of WEE1 as a potential molecular target in cancer cells by RNAi screening of the human tyrosine kinome. Breast Cancer Res Treat 122: 347-357, 2010.

45. Mir SE, De Witt Hamer PC, Krawczyk PM, Balaj L, Claes A, Niers JM, Van Tilborg AA, Zwinderman AH, Geerts D, Kaspers GJ, et al: In silico analysis of kinase expression identifies WEE1 as a gatekeeper against mitotic catastrophe in glioblastoma. Cancer Cell 18: 244-257, 2010. 